\title{
Fig. 57
}

(A)



(B)



Fig. S7. Integrin $\alpha_{v} \beta_{3}$, but not integrin $\beta_{1}$ involves in HSG-mediated RhoA activation. ECs were pretreated with integrins $\alpha_{\mathrm{v}} \beta_{3}$ and $\beta_{1}$ blocking antibodies $(10 \mu \mathrm{g} / \mathrm{ml})$ or IgG for $2 \mathrm{~h}$ prior to be seeded on HSG and LSG for $4 \mathrm{~h}$. Cell lysates were subjected to an RBD-pull down assay and detected with an antibody against RhoA. 